\section{Sincalide-Stimulated Cholescintigraphy: What Is the Standard?}

TO THE EDITOR: The article by Ziessman et al. adds to the further understanding of gallbladder physiology and the effect of cholecystokinin (CCK) stimulation (1). They suggest considering a $38 \%$ gallbladder ejection fraction (GBEF) as the lower limit of normal for a $0.02 \mu \mathrm{g} / \mathrm{kg}$ infusion of CCK-8 over $60 \mathrm{~min}$ and that their method of study should become the standard for routine clinical use. We would like to comment on some limitations inherent in the design, execution, and conclusions of their study that one should take into consideration in determining whether their method should become the standard.

Ziessman et al. chose a Food and Drug Administration-approved CCK-8 dose $(0.02 \mu \mathrm{g} / \mathrm{kg}$ to be given over $0.5-1 \mathrm{~min})$ but decided to infuse over 15,30 , or $60 \mathrm{~min}$, thus changing the dose rate at each level. The gallbladder continues to empty during and for 8-12 min after CCK-8 infusion. An accurate measurement of GBEF, therefore, requires that the downward slope of the curve be included in the calculation of GBEF. Drug dose-response studies require that either the dose rate or the duration of infusion be kept constant to test the complete effect of the drug. Because the GBEF does not follow a Gaussian distribution, one cannot use the mean and SD to set the lower limit of the reference range. The mean and wide SD of GBEF values shown in their Table 1 confirm the variability of GBEF seen in healthy subjects. Therefore, one needs to establish the frequency or distribution data to set the lower limit for a normal response. For the gallbladder phase of the study, we collect data at $1 \mathrm{frame} / \mathrm{min}$ for $30 \mathrm{~min}$; and beginning at $3 \mathrm{~min}$, CCK-8 is infused at a rate of $3 \mathrm{ng} / \mathrm{kg} / \mathrm{min}$ for $10 \mathrm{~min}$. A GBEF value below $50 \%$ is considered abnormal. Approximately 50\% of the patients with chronic acalculous or chronic calculous cholecystitis experience low- to moderate-intensity abdominal pain during or after CCK-8 infusion. The total duration of 90-min data collection allows diagnosis of various types of hepatobiliary and gastrointestinal diseases associated with bile formation and flow (2).

The study designed by Ziessman et al. is much longer (120 min) than our method ( $90 \mathrm{~min}$ ), and abrupt termination of CCK-8 data collection at $120 \mathrm{~min}$ results in missing the downward slope of the curve in the calculation of an accurate GBEF (2). Paradoxic gallbladder filling seen in sphincter-of-Oddi spasm occurs immediately after termination of the CCK-8 infusion and will be missed if data collection is not continued for an additional 10-15 min. In addition, $60 \mathrm{~min}$ of continuous CCK-8 infusion induces bile transit throughout the small intestine, often reaching the colon. This prevents detection of CCK-8-induced intestinal hyperperistalsis indicative of irritable bowel syndrome seen with a shorter CCK-8 infusion. Radiolabeled bile is an excellent tracer not only for measurement of GBEF but also for detection of various other hepatobiliary and gastrointestinal diseases (2). Most patients who experience abdominal pain and discomfort with CCK-8 are likely to prefer a 30-min infusion over $60 \mathrm{~min}$. The suggestion that their method of study should become the standard

COPYRIGHT ๑ 2010 by the Society of Nuclear Medicine, Inc. for routine clinical use may not be very appealing from the patient's point of view.

\section{REFERENCES}

1. Ziessman HA, Tulchinsky M, Lavely WC, et al. Sincalide-stimulated cholescintigraphy: a multicenter investigation to determine optimal infusion methodology and gallbladder ejection fraction normal values. J Nucl Med. 2010;51:277-281.

2. Krishnamurthy GT, Krishnamurthy S. Nuclear Hepatology: A Textbook of Hepatobiliary Diseases. 2nd ed. New York, NY: Springer; 2009:299-317.

\section{Gerbail T. Krishnamurthy* Shakuntala Krishnamurthy \\ *Tuality Community Hospital \\ 335 S.E. 8th Ave. \\ Hillsboro, OR 97123 \\ E-mail: gtkrishna@aol.com}

DOI: 10.2967/jnumed.110.076588

REPLY: The purpose of our investigation was to determine a sincalide infusion methodology that has the least variability (lowest coefficient of variation) by comparing 3 methods (15-, 30-, and 60-min infusions of $0.02 \mu \mathrm{g} / \mathrm{kg}$ ) and to establish reference values (1). The 3-min infusion method was not investigated because it has previously been shown to have variable and unpredictable results $(2,3)$. This sincalide investigation has the largest number of healthy subjects ever studied and is the first published multicenter study that established sincalide-stimulated gallbladder ejection fraction (GBEF) reference values, and no prior study has compared 3 different infusion methods in the same subjects. The statistical analysis is strong and confirms the significance of our results.

In their letter to the editor the writers state that they use a 10min cholecystokinin-8 infusion with imaging for 27 min, allowing them to capture gallbladder emptying that occurs after the infusion stops, which they consider important. That may be true for methods with short infusion times. Our 15-min infusion showed minor additional emptying after infusion cessation (Table 1). However, the 30-min infusion showed no additional emptying after the infusion ends. A similar lack of additional emptying after the end of infusion was reported by others for a 45-min infusion method in healthy subjects and patients (4). Therefore, imaging after the end of cholecystokinin-8 infusion may capture additional emptying when short infusion methods are used, but it adds nothing to the longer ( $\geq 30 \mathrm{~min}$ ) infusion methods.

The writers further state that they consider a GBEF value below $50 \%$ as abnormal. However, there are no published data to substantiate that claim. It is unclear, then, why these investigators continue to promote short infusions. Although they have published numerous papers claiming that less than $35 \%$ is abnormal for a 3-min infusion and less than $50 \%$ for a 10 -min infusion, they have published no healthy-subject data to substantiate that statement (5-8). The closest they come is in a 2002 publication about a study in which they initially studied 21 healthy subjects (6). However, 8 subjects were excluded because their 
GBEF was less than $35 \%$, leaving only 13 subjects. The investigators did not claim to have established reference values in that publication.

One might expect the letter writers' method to have results similar to our 15-min infusion. Table 1 of our article provides the mean GBEF and SD for a 15-min infusion, imaged for and quantified at 30,45 , and $60 \mathrm{~min}$. Our data strongly suggest that the authors' conclusion regarding reference values is not correct. From our data, the lower limits of normal for their method would more likely be less than $25 \%$ (fifth percentile). In regard to the statistical criticism, we clearly noted that the data were not Gaussian in distribution and that we therefore used percentiles rather than mean and SD to establish reference values.

The writers misinterpret the relationship between abdominal pain and sincalide infusion. No subject we studied had abdominal pain with a 30-min or 60-min infusion, although 2 subjects had symptoms with a 15-min infusion. Prior data have shown that the incidence of pain with a 3-min sincalide infusion approaches $50 \%$ (2). Thus, the incidence of abdominal pain in healthy subjects is seen only with short infusion times. In healthy controls and patients with gallbladder disease, Yap et al. found that none had abdominal pain with a 45-min infusion, including those with low GBEFs (4). This is another important reason to use the slow infusion method-the patients will appreciate it.

Referring clinicians have had 2 concerns about the use of sincalide cholescintigraphy to diagnose chronic acalculous gallbladder disease: the first is the lack of sufficient evidence-based data proving the diagnostic utility of the GBEF, and the second is the lack of standardization of the infusion methodology $(9,10)$. We strongly recommend general acceptance of the 60-min infusion method as the standard and that this methodology and these reference values become the standard. We hope that a prospective multicenter investigation will be initiated using this methodology with the expectation that it will confirm the utility of sincalide cholescintigraphy for selecting candidates for cholecystectomy.

\section{REFERENCES}

1. Ziessman HA, Tulchinsky M, Lavely WC, et al. Sincalide-stimulated cholescintigraphy: a multicenter investigation to determine optimal infusion methodology and gallbladder ejection fraction normal values. J Nucl Med. 2010;51:277-281.

2. Ziessman HA, Fahey FH, Hixson DJ. Calculation of a gallbladder ejection fraction: advantage of continuous sincalide infusion over the 3-min infusion method. J Nucl Med. 1992;33:537-541.

3. Ziessman HA, Muenz LR, Agarwal AK, ZaZa A. Normal values of sincalide cholescintigraphy: comparison of two methods. Radiology. 2001;221:404410.

4. Yap L, Wychereley AG, Morphett AD, et al. Acalculous biliary pain: cholecystectomy alleviates symptoms in patients with abnormal cholescintigraphy. Gastroenterology. 1991;101:786-793.

5. Krishnamurthy GT, Krishnamurthy S, Brown PH. Constancy and variability of gallbladder ejection fraction: impact on diagnosis and therapy. $\mathrm{J} \mathrm{Nucl} \mathrm{Med}$. 2004;45:1872-1877.

6. Krishnamurthy GT, Brown PH. Comparison of fatty meal and intravenous cholecystokinin infusion for gallbladder ejection fraction. $J$ Nucl Med. 2002;43:1603-1610.

7. Krishnamurthy GT, Krishnamurthy S. Diagnostic reliability of gallbladder ejection fraction. Indian J Nucl Med. 2002;17:13-17.

8. Krishnamurthy S, Ceruli-Switzer J, Chapman N, Krishnamurthy GT. Comparison of gallbladder ejection fraction obtained with regular CCK- 8 and pharmacy compounded CCK-8. J Nucl Med. 2003;44:499-504.

9. DiBaise JK, Oleynikov D. Does gallbladder ejection fraction predict outcome after cholecystectomy for suspected chronic acalculous gallbladder dysfunction? A systematic review. Am J Gastroenterol. 2003;98:2605-2611.
10. Delgado-Aros S, Cremonini F, Bredenoord AJ, Camilleri M. Systematic review and meta-analysis: does gall-bladder ejection fraction on cholecystokinin cholescintigraphy predict outcome after cholecystectomy in suspected functional biliary pain? Aliment Pharmacol Ther. 2003;18:167-174.

\author{
Harvey A. Ziessman* \\ Mark Tulchinsky \\ Alan H. Maurer \\ *Johns Hopkins Medical Institute \\ 601 N. Caroline St., Suite 3231 \\ Baltimore, MD 21278 \\ E-mail: hziessm1@jhmi.edu
}

DOI: 10.2967/jnumed.110.076646

\section{Validating PET Scanner Calibration for Multicenter Trials}

TO THE EDITOR: With interest we read the recent publication of Scheuermann et al. (1), who reported on the experience of the American College of Radiology Imaging Network in qualifying PET scanners to participate in multicenter trials. The network does so by analyzing submitted PET scans of uniform cylinders (either solid ${ }^{68} \mathrm{Ge}$ or fillable with ${ }^{18} \mathrm{~F}$ ) to verify the accuracy of scanner calibration (in terms of standardized uptake values) and by qualitatively reviewing typical patient images. Because many of the sites tested have been unable to produce acceptable results on the first attempt, the authors concluded that a verification of the basic scanner calibration is extremely important before sites can be allowed to participate in multicenter trials.

From our experience (2), we fully support this final conclusion. In particular, we agree that testing with fillable phantoms provides an independent check of system calibration and is a useful metric in characterizing the operator's experience in measuring and recording the injected dose accurately. The problems encountered are likely to occur in clinical acquisitions, too. The authors claim that using an identical phantom for calibration or normalization and for standardized uptake value testing, that is, a ${ }^{68} \mathrm{Ge}$ cylinder, may propagate errors. This claim is reflected in our findings, also. In our opinion, using the same phantom for calibration and verification is in some way a circular argument and may even completely hide calibration errors.

In the qualification process for PET scanners used in German multicenter trials, a somewhat different approach is followed (2), emphasizing testing of all equipment involved in the final analysis chain. Basically, each scanner is calibrated in terms of activity concentration, which is rescaled to standardized uptake values by normalization to the ratio of injected activity to body volume (approximated by patient weight). Therefore, careful cross calibration between PET scanner and dose calibrator is essential $(2,3)$. The verification chain therefore starts with the dose calibrator, whose accuracy is checked by certified ${ }^{68} \mathrm{Ge}$ sources. This test not only verified the instrument itself but also facilitated the identification of errors in the subsequent chain. The PET scanner calibration and processing is then tested through measurement of a cylindric phantom filled with a known activity concentration of ${ }^{18} \mathrm{~F}$ solution, relying on the accuracy of the calibrator. Data were acquired to a high statistical quality to facilitate the detection of systematic errors during subsequent analysis of reconstructed images. 